\title{
The Effect of Recombinant Human Growth Hormone Therapy in Patients with Completed Stroke: A Pilot Trial
}

\author{
Junyoung Song, M.D., Kicheol Park, M.D., Hakil Lee, M.D., Min Young Kim, M.D., Ph.D. \\ Department of Rehabilitation Medicine, CHA University, Seongnam 463-712, Korea
}

\begin{abstract}
Objective To evaluate the safety and potential efficacy of "recombinant human growth hormone (rhGH)" on the functional recovery of completed stroke patients.

Method Completed stroke patients were recruited. All participants were randomly assigned to the GH group (rhGH injection and rehabilitative therapy) or the control group (only rehabilitative therapy). Above all, they were closely monitored for safety. Further, for the efficacy measurement, Korean Modified Barthel Index (K-MBI), Manual Muscle strength Test (MMT), and Fugl-Meyer assessment (FMA) were assessed to determine the changes of functional recovery during 6-months of the study period. Along with it, diffusion tensor image was taken as the baseline, and a followed-up study to observe the changes in diffusion tensor tractography (DTT), during the period, and one patient in the GH group was also examined with functional MRI (fMRI). Index of fatigue on 5 point scale for the study period was also assessed.

Results Twenty-two patients were enrolled, and 15 completed the study and were included in the analysis. No harmful adverse events were observed in the GH group. By comparison between the groups, the GH group showed more improvement in K-MBI than the control group ( $p<0.05)$. DTT showed less decrement of fibers in the GH group than in the control group, without statistical significance. fMRI showed an increment in the activated area. Patients in the GH group expressed no fatigue at all, during the study period $(\mathrm{p}=0.00)$.

Conclusion The administration of rhGH in long term resulted in the improvement in K-MBI, and subjectively less tiredness during the injection period.
\end{abstract}

Key Words Stroke, Growth hormone, IGF-1, Recovery

Received February 22, 2012; Accepted May 21, 2012

Corresponding author: MinYoung Kim

Department of Rehabilitation Medicine, CHA Bundang Medical Center,

CHA University, 59, Yatap-ro, Bundang-gu, Seongnam 463-712, Korea

Tel: +82-31-780-6281, Fax: +82-31-780-3449, E-mail: kmin@cha.ac.kr

(a) This is an open-access article distributed under the terms of the Creative Commons Attribution Non-Commercial License (http:// creativecommons.org/licenses/by-nc/3.0) which permits unrestricted noncommercial use, distribution, and reproduction in any medium, provided the original work is properly cited.

Copyright $\odot 2012$ by Korean Academy of Rehabilitation Medicine

\section{INTRODUCTION}

Over the past decades, it was not expected that the stabilized brain damage, including the completed stroke, ${ }^{1}$ could be significantly recovered. However, many basic and clinical experiments revealed the existence of recovery potential after brain damage. ${ }^{2,3}$ The kernel mechanism of substantial recovery is called "brain plasticity". Brain plasticity encompasses all possible mechanisms of 
neuronal reorganization: recruitment of pathways that are functionally homologous to, but anatomically distinct from the damaged ones; synaptogenesis; dendritic arborization; and reinforcement of existing, but functionally silent synaptic connections. ${ }^{4,5}$ Above all, the fundamental brain plasticity would be neurogenesis. The persistence of neurogenesis in the forebrain subventricular zone of adult mammalians suggests that the mature brain maintains the potential for neuronal replacement after injury. ${ }^{6}$

'Neurotrophic factors,' including several mitogens, growth factors, cytokines and cell division modulators were found to mediate the activity of neurogenesis and angiogenesis, and regulates gene expressions involving the neurogenesis. ${ }^{7-9}$ Many factors, such as brain-derived neurotrophic factor, ciliary neurotrophic factor, NT-3/4, glial cell-derived neurotrophic factor, and vascular endothelial growth factor, are examples. ${ }^{10}$

Among the neurotrophic factors, insulin-like growth factor- 1 (IGF- 1$)^{11}$ is a representative one. In human, the serum IGF-I level was found to be depressed, following an acute stroke, ${ }^{12}$ while the brain IGF-I level in the vicinity of the lesion, was found to be elevated in rodent models of stroke injury. ${ }^{13}$ These changes might reflect an autonomous protective response. ${ }^{14}$ In the brain, IGF1 seemed to be involved in many mechanisms, showing potentiality of therapeutic usage. First, IGF-1 promotes neurogenesis. ${ }^{15}$ A study that experimented the effects of IGF-1, by overexpression and ablation of the components of the IGF system, indicated pivotial role of IGF-1 in brain development by not only stimulating the neurogenesis, but also synaptogenesis, oligodendrocyte development, and promoting the survival of them. ${ }^{16}$ Second, IGF-1 augmentes synaptogenesis. IGF-1 overexpression produced persistent increases in the total number of synapses in the dentate gyrus. ${ }^{15}$ Third, IGF-1 has anti-apoptotic effects and protected neurons against various conditions, such as excitotoxicity and oxidative stress, glutamate, nitric oxide, and hydrogen peroxide-induced apoptosis. ${ }^{17-19}$ IGF-1 also protects the oligodendrocyte precursors from cytotoxicity ${ }^{20}$ and mature oligodendrocytes from the death-inducing effects of tumor necrosis factor- $\alpha{ }^{21}$ Fourth, IGF-1 promotes proliferation and maturation of cells in oligodendrocyte lineage and stimulate mylelination. ${ }^{16}$ Indeed, animal experiments showed beneficial effects of exogenous IGF-I after stroke by showing a reduction of infarction volume and facilitation of neurog- nesis. $^{22}$

However, clinical use of IGF- 1 is not established because of safety issues. ${ }^{23}$ IGF-1 was known to exacerbate carcinogenesis and its high concentration denoted increased cancer risk. ${ }^{24}$ Another concern is disrupting the growth hormone homeostasis for the IGF-1, which is the end product of growth hormone pituitary axis. ${ }^{25}$ Thus, instead of IGF-1, recombinant human growth hormone (rhGH) might be considered, as it has been used without a safety problem in the clinical field. It was reported that rhGH administration for six months to normal adults with diminished growth hormone secretion caused an increase in the serum IGF-1 concentrations. ${ }^{26}$ Accumulation of systematic IGF-1 can elevate the level of IGF-1 at the injury site. ${ }^{27,28}$ If exogenous administration of rhGH elevates IGF-1 concentration, at the injury site, then it might enhance the therapeutic brain plasticity, and might result in functional improvement.

Even though it was not available to confirm brain plasticity directly in the human brain, we can indirectly demonstrate it by brain imaging studies. The diffusion tensor imaging (DTI ${ }^{29}$ of the brain and the functional magnetic resonance imaging (fMRI) ${ }^{30,31}$ are the representative methods to find the reorganization process.

Through the present pilot study, we wanted to evaluate the clinical usability of rhGH. Thus, the first aim was to assess the harmful effect, and secondly, efficacy for functional recovery in completed stroke patients.

\section{MATERIALS AND METHODS}

This study was approved by the Institutional Review Board of Bundang CHA Medical Center, Republic of Korea.

\section{Subjects}

The present study included completed stoke patients who were receiving intensive rehabilitation treatment from Sep 2008 to Oct 2009. All patients were evaluated with routine physical examinations and thorough neurological examinations. The subjects were randomly assigned to one of the two groups, with a distribution ratio of $2: 3$; the rhGH study group (GH group) who received rhGH and the control group who received rehabilitation only. The study proceeded as open-labeled, as this was the first trial of GH therapy for stroke patients, and thus, 
the safety was not yet known for this purpose. The study began after all patients and their families signed the informed consent form.

Inclusion criteria were as follows: 1) completed stoke patients at least two month or more after the onset of cerebral infarction or hemorrhage; 2) having definite hemiplegia with disability as modified Rankin scale score 3 to 4 ; 3) serum IGF-1 levels lower than $300 \mathrm{ng} / \mathrm{ml}$; and 4) who had enough mental function and communication ability to follow the instructions of the therapy team. The exclusion criteria were: 1 ) poorly controlled diabetes patients because rhGH could lead to hyperglycemia, ketosis, and insulin resistance status; 2) patients with high blood pressure as greater than $150 \mathrm{mmHg}$ in systolic pressure, in spite of antihypertensive medication; and 3) patients with high tumor marker levels of AFP, CEA, CA 15-3, CA 19-9, and PSA for men, and AFP, CEA, CA 153 , CA 19-9, and CA-125 for women. Twenty-two patients were enrolled and 8 patients $($ male $=2$, female $=6$ ) were assigned in the GH group and 14 patients (male=9, female $=5$ ) were assigned in the control group.

\section{Rehabilitation}

All participated patients received a comprehensive rehabilitation treatment, including twice a day of physical therapy, which included muscle strengthening exercise, gait training, neurodevelopmental treatment, and twice a day of occupational therapy, which included cognitive training, activity of daily living training, upper extremities muscle exercise everyday, 30 minutes at a time during their hospitalization that were continued more than 2 months. After discharge, all the patients underwent rehabilitation treatment, including one time of physical therapy and occupational therapy, which lasted 30 minutes at a time for more than 3 days a week, until the completion of this study, about 6 months after each enrollment.

\section{Recombinant human growth hormone (Declage ${ }^{\circledR}$ ) therapy}

Declage $^{\circledR}$ (LG Life Sciences Ltd., Iksan, Korea) was used for this trial. It was subcutaneously injected with $3 \mathrm{mg}$ a week in the GH group for the duration of 24 weeks. After discharge, GH group were received Declage ${ }^{\circledR}$ weekly by a nurse in the injection room. Serum 1GF-1 levels were measured at an interval of 2 months, for a total of three times during the treatment period.

\section{Assessment for harmful effect of rhGH}

During the whole period of the study, all the subjects in the GH group were guided to report any adverse events. In the $\mathrm{GH}$ group, $\mathrm{CBC}$ with differential cell count, serum glucose, BUN, Creatinine, electrolytes ( $\mathrm{Na}, \mathrm{K}, \mathrm{Cl}$ ) levels were checked monthly. Glucose level was checked after an overnight fasting. Vital signs, including blood pressure and heart rate, were assessed daily during hospitalization and weekly after discharge when they visited for rehabilitation.

\section{Functional evaluations}

All patients were assessed for functional measurements, including Korean modified Barthel index (K-MBI), Manual Muscle strength Test (MMT), Fugl-Meyer assessment of the upper extremity (FMA), and Korean version of the Mini-Mental Status Examination (K-MMSE). Representative scores of MMT from six muscle groups, in affected limb, shoulder flexor, elbow flexor, 3rd finger proximal interphalangeal flexor, hip flexor, knee extensor, and ankle dorsiflexor of affected extremities were selected for summated score in five grades (Zero $=0$, Trace $=1$, Poor $=2$, Fair=3, Good=4, Normal=5), which made the total score of 30 .

\section{Fatigue scale}

All the patients was asked about their subjective feeling of fatigue, compared to the usual status of interval from the onset of stroke to the study participation, and the usual status of the study period, according to a fatigue scale, which contained 5 degree of fatigue status. It was made to choose a number from -2 to +2 , depending on their feeling of fatigue. The degree of -2 meant definitely more tired, while +2 degree meant definitely less tired. In the control group, 2 patients reported -1 degree, which meant somewhat less tired, 4 patients 0 degree which meant tired as usual status and 5 patients reported +1 degree, which meant somewhat less tired. However, in the GH group, all the patients reported +2 degree.

\section{Brain imaging study}

All patients underwent a routine brain MRI, using 1.5 MAGNETOM Sonata (Simens, Berlin, Germarny) or 3T GE Signa System (General-Electric, Milwaukee, USA). DTI data were also acquired, using a 2D axial spin echo-planar imaging with refocusing pulses. The sequence parameters 
were: a TR/TE of 12000/108 msec; 1NEX, 48 slices; $24 \mathrm{~cm}$ FOV; 128 $\times 128$ matrix; $3.0 \mathrm{~mm}$ slice thickness; 25 gradient directions; $\mathrm{B}=900$; with a non-diffusion weighted baseline image $(\mathrm{B}=0)$. All patients in the $\mathrm{GH}$ group and 4 patients in the control group underwent a DTI follow-up study, at 6 months later. We evaluated the fiber connectivity using a 3D fiber reconstruction algorithm from the fibers that pass the whole area of midbrain. Diffusion tensor tractography (DTT), as a result of reconstruction, was officially reported by a radiologist. Further, the changes of DTT were expressed as no change, minimal and obvious increase or decrease in fibers.

One patient in the GH group underwent fMRI at the enrollment to this study, and was followed up 6 months later. Twice of fMRI were performed on a 3T General Electric scanner with blood oxygenation level, and dependent images were obtained using an axial single-shot gradient echo planar imaging sequence with a resolution of $64 \times 64$ and a slice thickness of $5 \mathrm{~mm}$ to cover the volume of $220 \times 220 \times 150$ $\mathrm{mm}^{3}$ with a TE/TR=320/3000 $\mathrm{ms}$ and a flip angle of $90^{\circ}$. A zscore threshold of 7.5 was chosen to identify active regions. The fMRIs consisted of runs of $30 \mathrm{~s}$ of rest, followed by $30 \mathrm{~s}$ of activity, repeated three times. The runs were as follows:
1) motor, affected hand grasp, 2) motor, unaffected hand grasp, and 3) motor, bilateral hand grasp. Before the fMRI, the patient practiced hand grasp movement by a verbal command. The images were reconstructed and motion corrected, and the correlation coefficient between the ideal activation/rest time course and the measured data was calculated for each voxel in the image. Finally, the data were overlaid onto $\mathrm{T} 1$ anatomical images $(\mathrm{z}=7.5)$.

\section{Statistical analysis}

Wilcoxon signed-rank test was performed for assessing the changes in the measured parameters, during 6 months of treatment in each group, and Mann-Whitney $\mathrm{U}$ test was used to compare the outcomes between the groups. Fisher's exact test was used to see the difference in fatigue score. For the analyses, Statistical Package for the Social Sciences (SPSS) version 19.0 program in Bundang CHA Medical Center was used.

\section{RESULTS}

\section{Subjects}

Before the completion of this study, 7 patients dropped

Table 1. The Characteristics and Baseline Insulin-like Growth Factor-1 of GH Group and Control Group

\begin{tabular}{|c|c|c|c|c|}
\hline Group & Sex/Age (year) & Diagnosis & Time interval after onset (days) & IGF-1 (ng/ml) \\
\hline \multicolumn{5}{|l|}{ GH group } \\
\hline $\mathrm{Al}$ & $\mathrm{F} / 67$ & Rt. BG* \& $\mathrm{PVWM}^{\dagger}$ infarction & 83 & 78.4 \\
\hline A2 & $\mathrm{F} / 63$ & Rt. BG infarction & 123 & 121.0 \\
\hline A3 & $\mathrm{F} / 60$ & Rt. BG $\mathrm{ICH}^{+}$ & 58 & 57.8 \\
\hline A4 & $\mathrm{M} / 55$ & Lt. Temporal ICH & 237 & 81.8 \\
\hline A5 & $\mathrm{M} / 72$ & Lt. Thalamus \& BG ICH & 327 & 131.0 \\
\hline \multicolumn{5}{|c|}{ Control group } \\
\hline $\mathrm{B} 1$ & $\mathrm{~F} / 64$ & Rt. BG infarction & 178 & 108.0 \\
\hline B2 & $\mathrm{M} / 61$ & Rt. BG ICH & 65 & 146.0 \\
\hline B3 & $\mathrm{M} / 73$ & Rt. $\mathrm{MCA}^{\S}$ infarction & 185 & 78.5 \\
\hline B4 & $\mathrm{M} / 77$ & Rt. MCA infarction & 69 & 73.0 \\
\hline B5 & $\mathrm{F} / 73$ & Rt. BG infarction & 141 & 67.9 \\
\hline B6 & $\mathrm{M} / 66$ & Rt. BG infarction & 224 & 273.0 \\
\hline B7 & $\mathrm{M} / 74$ & Lt. BG infarction & 87 & 91.4 \\
\hline B8 & $\mathrm{M} / 41$ & Lt. BG ICH & 166 & 207 \\
\hline B9 & $\mathrm{M} / 60$ & Rt. Thalamus ICH & 90 & 172.0 \\
\hline B10 & $\mathrm{F} / 63$ & Lt. MCA infarction & 147 & 176.0 \\
\hline
\end{tabular}

A: GH group, B: Control group

${ }^{*}$ BG: Basal ganglia, ${ }^{\dagger} \mathrm{PVWM}$ : Periventricular white matter, ${ }^{\dagger} \mathrm{ICH}$ : Intracerebral hemorrhage, ${ }^{\circledR} \mathrm{MCA}$ : Middle cerebral artery 
Table 2. Raw Data of GH Group and Control Group

\begin{tabular}{|c|c|c|c|c|c|c|c|c|}
\hline Group & $\begin{array}{c}\text { K-MBI* } \\
\left.\text { (Baseline }^{\dagger}\right)\end{array}$ & $\begin{array}{c}\text { K-MBI* } \\
\left.\text { (End point }^{\dagger}\right)\end{array}$ & $\begin{array}{c}\text { FMT }^{\mathfrak{S}} \\
\text { (Baseline) }\end{array}$ & $\begin{array}{c}\text { FMT } \\
\text { (End point) }\end{array}$ & $\begin{array}{l}\text { K-MMSE }{ }^{\|} \\
\text {(Baseline) }\end{array}$ & $\begin{array}{c}\text { K-MMSE } \\
\text { (End point) }\end{array}$ & $\begin{array}{c}\text { MMT }^{\mathrm{T}} \\
\text { (Baseline) }\end{array}$ & $\begin{array}{c}\text { MMT } \\
\text { (End point) }\end{array}$ \\
\hline \multicolumn{9}{|l|}{ GH group } \\
\hline Al & 74 & 94 & 41 & 60 & 23 & 25 & 15 & 20 \\
\hline $\mathrm{A} 2$ & 89 & 95 & 59 & 60 & 30 & 26 & 22 & 22 \\
\hline A3 & 36 & 74 & 13 & 22 & 24 & 27 & 4 & 14 \\
\hline $\mathrm{A} 4$ & 26 & 61 & 22 & 38 & 22 & 22 & 16 & 17 \\
\hline A5 & 31 & 51 & 4 & 13 & 16 & 17 & 8 & 8 \\
\hline \multicolumn{9}{|c|}{ Control group } \\
\hline B1 & 80 & 90 & 16 & 23 & 22 & 26 & 13 & 15 \\
\hline $\mathrm{B} 2$ & 35 & 43 & 12 & 14 & 30 & 30 & 0 & 7 \\
\hline B3 & 69 & 74 & 41 & 66 & 25 & 21 & 18 & 23 \\
\hline B4 & 48 & 48 & 4 & 10 & 22 & 22 & 10 & 14 \\
\hline B5 & 0 & 4 & 4 & 4 & 7 & 4 & 6 & 6 \\
\hline B6 & 98 & 98 & 37 & 27 & 30 & 29 & 16 & 12 \\
\hline B7 & 6 & 6 & 6 & 6 & 15 & 15 & 10 & 9 \\
\hline B8 & 75 & 80 & 19 & 26 & 18 & 21 & 8 & 15 \\
\hline B9 & 87 & 92 & 47 & 56 & 29 & 28 & 18 & 18 \\
\hline $\mathrm{B} 10$ & 28 & 52 & 6 & 8 & 23 & 29 & 12 & 14 \\
\hline
\end{tabular}

${ }^{*} \mathrm{~K}$-MBI: Korean modified barthel index (K-MBI), ${ }^{\dagger}$ Baseline: Raw data at baseline, ${ }^{*}$ End point: Raw data at end point, ${ }^{\varsigma}$ FMT: Fugl-meyer test of upper extremity, "K-MMSE: Korean version of the Mini-mental status examination, "MMT: Representative scores of Manual muscle strength test (MMT) from six muscle groups: shoulder flexor; elbow flexor; 3rd finger proximal interphalangeal flexor; hip flexor; knee extensor; and ankle dorsiflexor in five grades (Zero=0, Trace=1, Poor=2, Fair=3, Good=4, Normal=5), that made the full score, 30 (affected extremities)

out as they did not participate in the follow-up assessments ( 3 patients in GH group, 4 patients in control group). Thus, 5 patients ( 2 men, 3 women) in the GH group and 10 patients ( 7 men, 3 women) in the control group were analyzed. The average age of all patients was $62.60 \pm 9.09$ years (range: $41-77$ years). At the recruitment, the duration after the first cerebral infarction or cerebral hemorrhage was $145.33 \pm 76.27$ days. Patients were hospitalized for $99.00 \pm 36.75$ days (GH group: $117 \pm 49.44$ days, control group: $88.78 \pm 25.37$ days, $\mathrm{p}=0.298$ ). All patients underwent rehabilitation of more than 3 days a week, until the completion of this study.

The baseline characteristics and IGF-1 levels of the GH group and the control group were shown in Table 1. The raw data of the GH group and the control group were shown in Table 2. Any differences of age and duration after the onset were not statistically presented. The scores of the functional evaluations, as the baseline assessment, did not show any differences between the GH group and the control group (Table 2). Declage ${ }^{\bullet}$ was injected in the

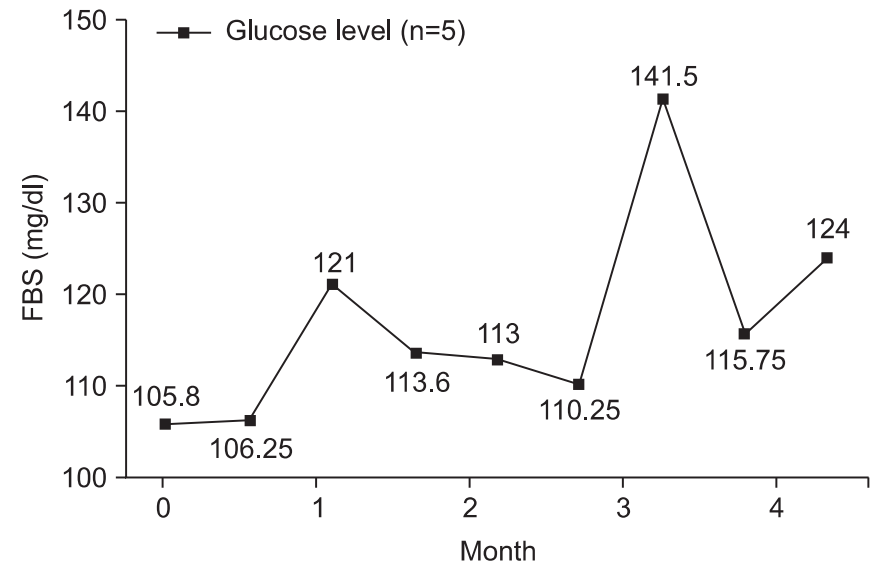

Fig. 1. Changes of glucose levels of the GH group. In the GH group, glucose levels were all within normal ranges during the rhGH therapy. FBS: Fasting blood sugar.

GH group at an average of 20.6 trials (range: 18-22 injections). 


\section{Adverse events}

Any adverse effects had not been observed during the study period, and up to now, at least 3 years after their enrollment in the GH group patients. We checked CBC with differential cell count, serum glucose, BUN, Creatinine, and electrolytes ( $\mathrm{Na}, \mathrm{K}, \mathrm{Cl}$ ) levels of the $\mathrm{GH}$ group 7.8 \pm 2.95 times (range: $3-11$ ). The values of CBC with dif- ferential cell count, serum glucose, BUN, Creatinine, and electrolytes $(\mathrm{Na}, \mathrm{K}, \mathrm{Cl})$ levels in the $\mathrm{GH}$ group were all within its normal range. Glucose levels were all within its normal range (Fig. 1). Vital signs, including blood pressure and heart rate, were not significantly changed by rhGH administration.
(A)

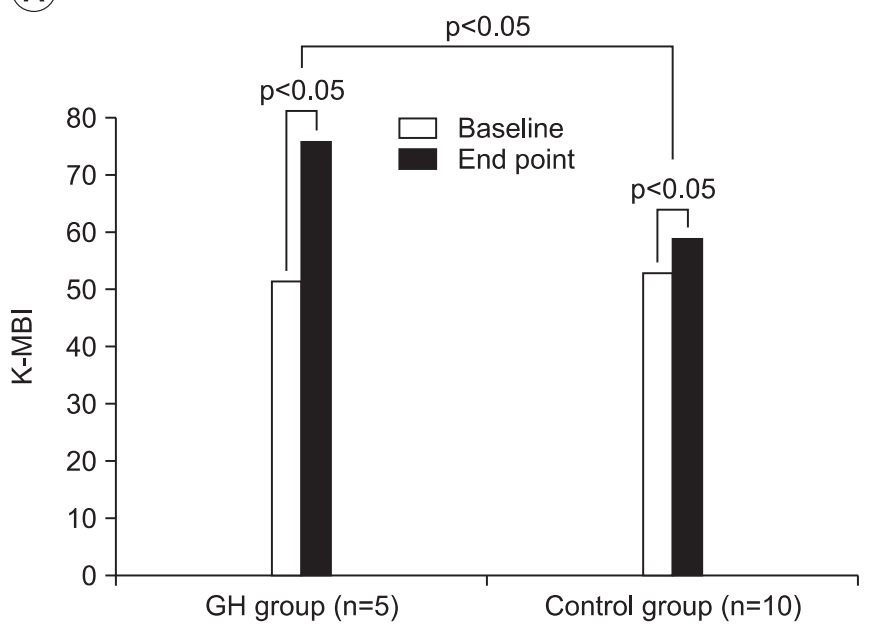

(C)

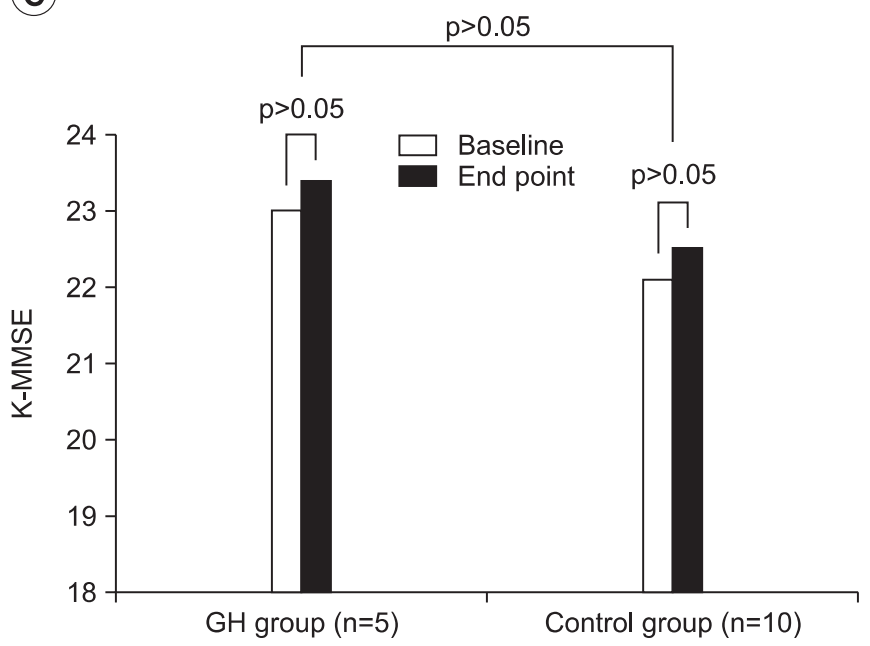

(B)

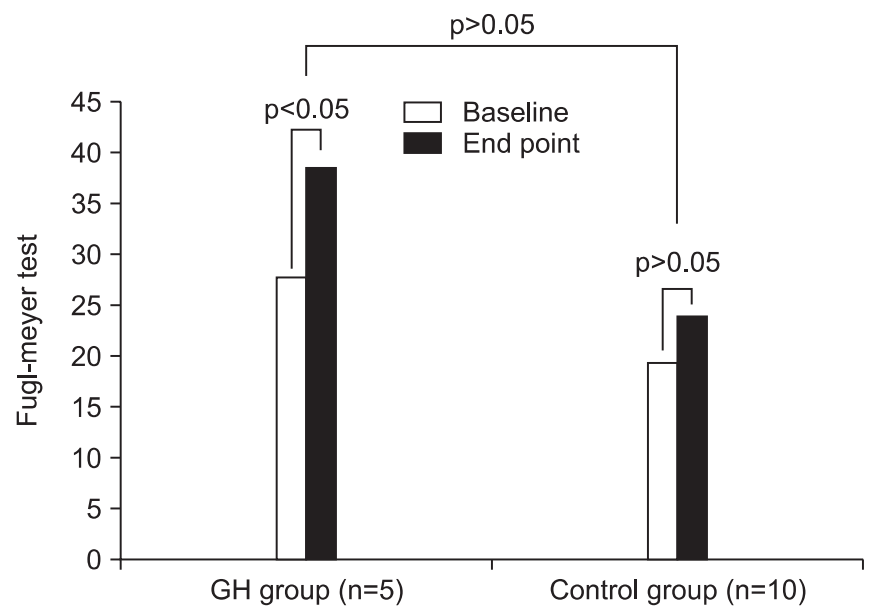

(D)

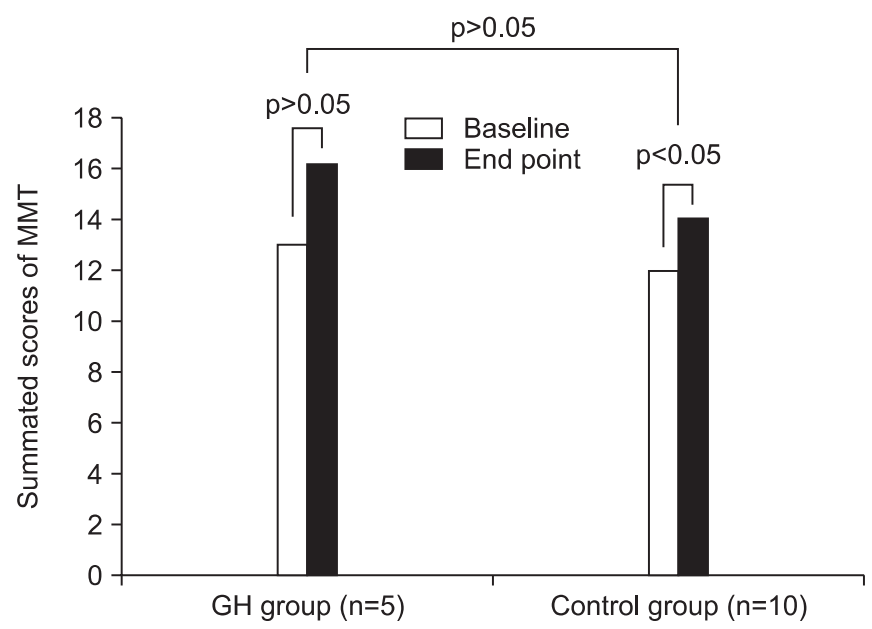

Fig. 2. Comparison of the changes in functional outcome within and between each group. (A) The GH group and the control group showed an increment of scores in K-MBI during 6 months of study period $(\mathrm{p}<0.05)$. The GH group showed more increment in the K-MBI score than the control group $(\mathrm{p}<0.05)$. (B) The GH group showed increment of scores in Fugl-meyer test of upper extremity during 6 months of study period $(\mathrm{p}<0.05)$. The control group did not show any difference during 6 months of the study period. (C) Both groups did not show any difference in the K-MMSE during 6 months of the study period. (D) The control group showed an increment of scores in the summated scores of MMT of the affected extremity during 6 months of the study period $(\mathrm{p}<0.05)$, however the GH group did not show any difference during the study period. K-MBI: Korean modified barthel index (K-MBI), K-MMSE: Korean version of the mini-mental status examination, MMT: Representative scores of manual muscle strength test (MMT) from six muscle groups: shoulder flexor; elbow flexor; 3rd finger proximal interphalangeal flexor; hip flexor; knee extensor; and ankle dorsiflexor in five grades (Zero=0, Trace=1, Poor=2, Fair=3, Good=4, Normal=5), that made the full score, 30 (affected extremities). 


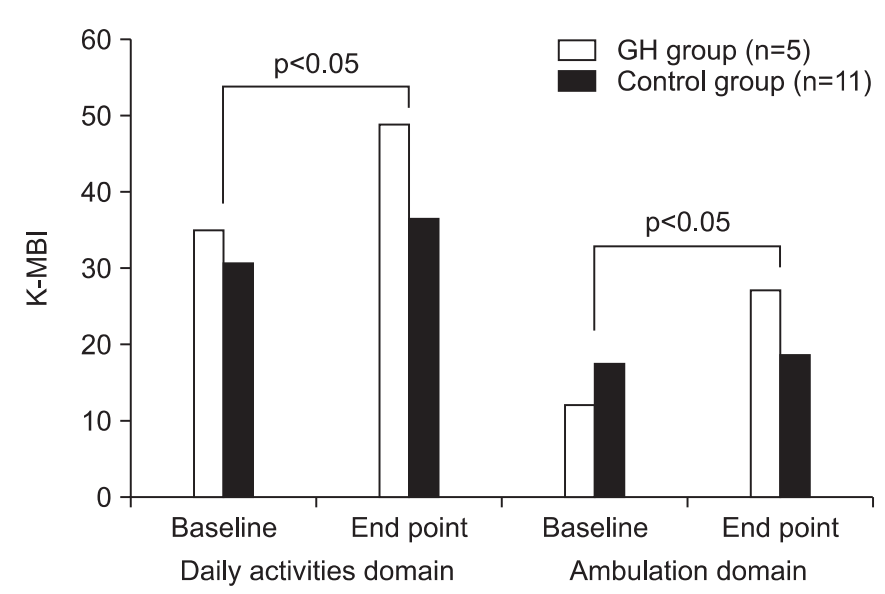

Fig. 3. Comparisons of changes of K-MBI between the GH group and the control group. When K-MBI was divided into the daily activity domain (personal hygiene, bathing, toilet, feeding, dressing, bowel and bladder control) and the ambulation domain (ambulation, stair climbing, chair/bed transfer), both showed increased K-MBI scores in each domain, the GH group showed more increment than the control group in the ambulation domain. K-MBI: Korean modified barthel index (K-MBI).

Changes of changes in functional outcomes within and between each group

In the GH group, the scores of the mean K-MBI and mean FMA were increased during the 6 months of the study period $(\mathrm{p}<0.05)$. In the control group, the mean scores of K-MBI and MMT summated score were increased, during the 6 months of the study period $(\mathrm{p}<0.05)$ (Fig. 2).

Comparison of changes of K-MBI score between two groups

The mean scores of K-MBI were increased in both groups by an intra-group analysis. However, the GH group showed more increment of the K-MBI mean score than the control group $(\mathrm{p}<0.05)$. For further analysis, the K-MBI was divided into daily activity domain (personal hygiene, bathing, toilet, feeding, dressing, bowel and bladder control) and ambulation domain (ambulation, stair climbing, chair/bed transfer). As a result, although both the GH group and the control group showed an increase in the K-MBI mean scores in each domain, the GH group showed more increment than that of the control group in the ambulation domain (Fig. 3).

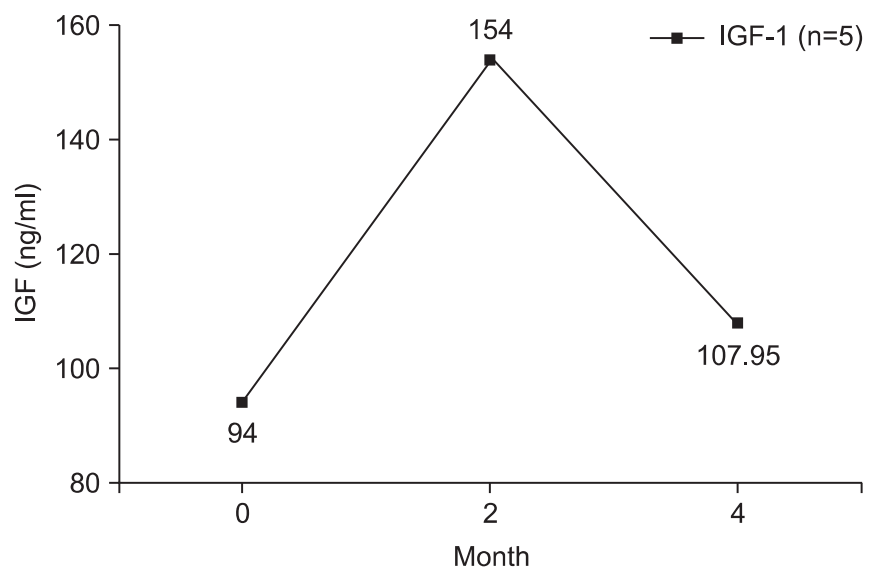

Fig. 4. Changes of IGF-1 levels of GH group $(n=5)$.

\section{Changes in IGF-1 level}

Serum IGF-1 was measured three times in the GH group (Fig. 4). The mean IGF-1 level was elevated at two months later, and receded at four months later. However, the mean IGF-1 level at four months was still higher than the initial IGF-1 level.

\section{Fatigue scale}

All the GH group patients reported +2 , which indicated a far less tiredness during the study period, compared to the control group $(\mathrm{p}=0.00)$. Further, they also reported that the subjects hardly caught a common cold or sickness during the rhGH injected study period.

Changes in diffusion tensor tractography and functional MRI

By the observation for changes in DTT in the GH group, 3 patients showed an obvious increase of the bilateral frontal fibers; 1 patient showed a mild increase; and 1 patient showed an obvious decrease of fibers (Fig. 5-A) In the control group, 1 patient showed minimal increase; 1 patient showed minimal decrease; and 2 patients showed obvious decrease of fibers (Fig. 5-B).

One patient who conducted an fMRI showed significant increased area of activation in the left motor cortex, compared to the previous fMRI study. He showed an increase in the grasp power of the affected hand, from $1 \mathrm{~kg}$ to $4 \mathrm{~kg}$ and K-MBI score from 25 to 61.

\section{DISCUSSION}

As this study result, GH group showed more of an im- 

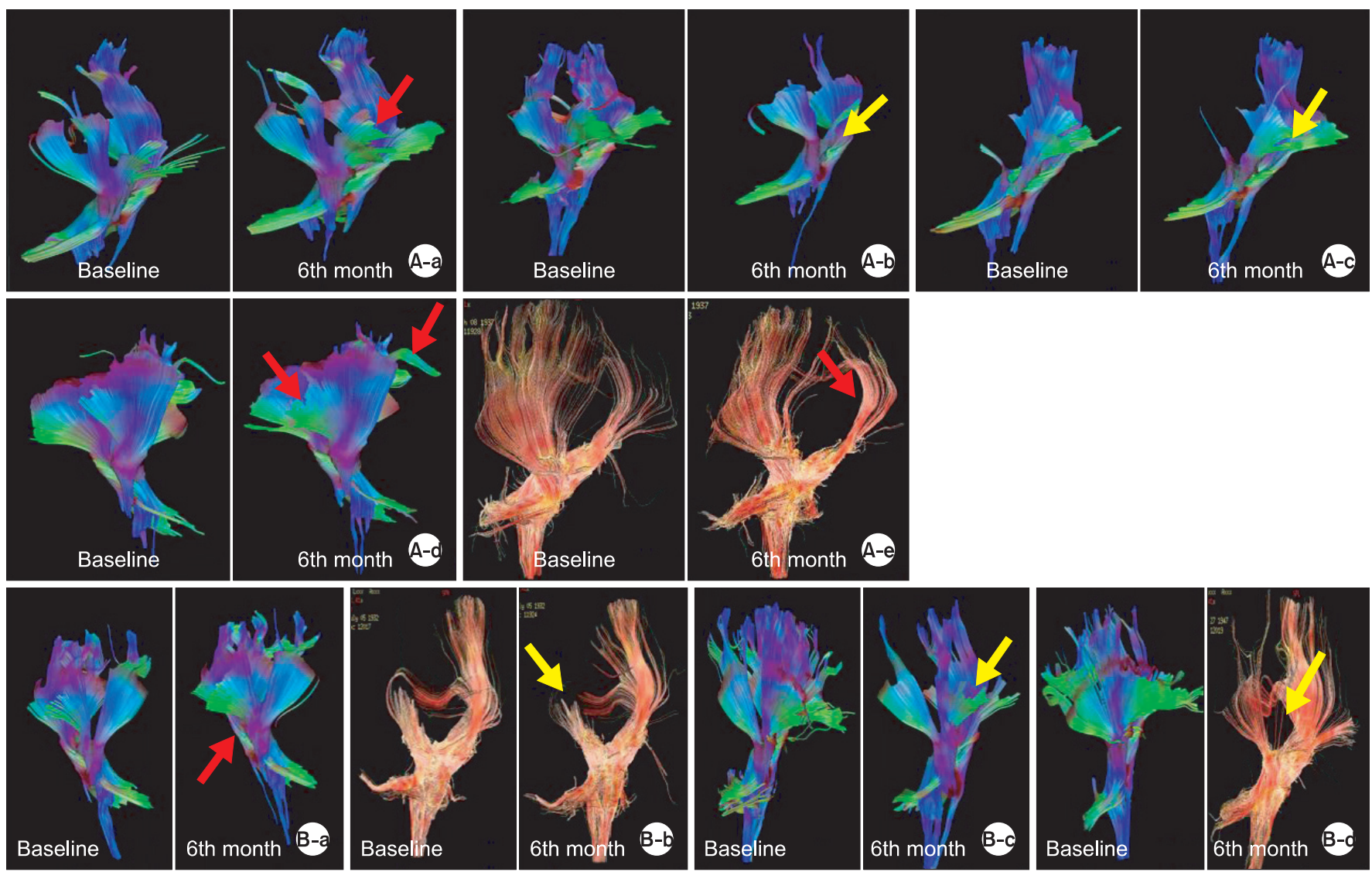

Fig. 5. Changes in DTT of GH group and control group. (A-a) DTT change in Al patient; increased fibers in bilateral frontal areas at 6 months compared to the baseline study. (A-b) DTT change in A2 patient; decreased fibers in bilateral frontal areas at 6 months, compared to the baseline study. (A-c) DTT change in A3 patient; decreased fibers in the left frontal areas at 6 months compared to the baseline study. (A-d) DTT change in A4 patient; increased fibers in the left frontal and posterior parietal areas at 6 months compared to the baseline study. (A-e) DTT change in A5 patient; mild increased fibers in the left parietal areas at 6 months compared to the baseline study. (B-a) DTT change in B1 patient; mild increased fibers in the left frontal and parietal areas at 6 months compared to the baseline study. (B-b) DTT change in B4 patient; mild decreased fibers in the right parietal areas and corpus callosum at 4 months compared to the baseline study. (B-C) DTT change in B6 patient; obvious decreased fibers in the right frontal areas at 4 months compared to the baseline study. (B-d) DTT change in B9 patient; obvious decreased fibers in the right frontal and parietal areas at 1 year compared to the baseline study. Red arrows: increased fibers, yellow arrow: decreased fibers.

provement in the K-MBI score, and felt less tired than the control group, without any sign of harmful effects. The growth hormone, that is synthesized, stored, and secreted from the somatotroph in the anterior pituitary gland, is known to promote improvements in the body composition, including an increase of muscle mass, reduction of total body fat, improvement of skin elasticity, and reduction in the rate of bone demineralization. ${ }^{32}$ It was also known to improve muscle strength, exercise performance, and more recently, to have anti-aging effect. ${ }^{33,34}$ Hence, the drug has been used as a supportive measure for the old.
The main objective of the present study was to investigate whether the use of rhGH would be helpful for functional recovery in completed stroke patients. Since we hypothesized that rhGH administration would stimulate the secretion of serum IGF-1 levels, which results in an elevation of the brain IGF-1 concentration, which could exert beneficial effect for the injured brain tissues. The results in the present study showed therapeutic possibility of rhGH (Declage ${ }^{\circledR}$ ) in completed stroke by showing a more increment in the score of K-MBI. The rhGH group showed an elevated score of FMA, by an intra-group analysis, but no superior outcome in MMT score or K- 
MMSE compared to the control group. These results did not seem to show definite efficacy of rhGH in the neurological recovery. However, as the improvement in daily activity of living would be an ultimate goal of functional gain for the disabled stroke patients, it could be interpreted as being significant. The better outcome in K-MBI could have come from the general effect of growth hormones, such as improvement of exercise performance. ${ }^{26}$ The patients in the GH group reported definitely less fatigue than the control group. Even though it is difficult to enlighten the mechanism, it could be assumed that rhGH provided better opportunity of participating rehabilitation treatment, by reducing fatigue.

Specifically, the beneficial effect on the injured brain tissues by rhGH was also expected. Studies with stroke animal model demonstrated a significant improvement in exercise function and spatial memory, when growth hormone was injected continuously for 6 weeks through the lateral part of cerebral ventricle, where an injected growth hormone was localized and converged at the ipsilateral part of SVZ, white matter, the injured brain region and the border area. ${ }^{35}$

To discuss about the effect GH on the brain, it is essential to consider the actions of IGF-1. In previous study, serum IGF-1 level was shown to increase in the proportion to the size of infarction in humans, suggesting that the serum IGF-1 level should be lowered in patients with stoke. ${ }^{28}$ However, recent studies have proposed that the serum IGF-1 levels, assessed within 24 hours of the onset of stroke, were significantly lower than its levels in the controls. Furthermore, low levels were related to poor outcomres, especially death. The higher the serum IGF1 levels, the better the outcome in its exercise capacity and recovery of the damaged tissue ${ }^{36,37}$ Degradation of the cognitive function occurred with the reduced levels of IGF-1 and the damaged hippocampal neurogenesis in streptozocin induced diabetic rats. ${ }^{38}$ In addition, administration of IGF-1 to diabetic rats appeared to prevent cognitive function decline. ${ }^{37}$ Another report proposed therapeutic expression of IGF-1 in microglia by a treatment for Alzheimer's disease. ${ }^{39}$ The overexpression of IGF-1 in stroke reduced symptom of paralysis and brought functional improvement. ${ }^{37}$ These results implied the contribution of IGF-1 for recovery mechanism of the injured brain. The IGF-1 level in the GH group was elevated at 2 months after commencement of rhGH admin- istration. However, the elevated level was not maintained until yjr fourth month, yet the level was higher than the baseline at the fourth month. Probably, the cause of failure in maintaining a high IGF-1 level was GH-IGF axis adaptation. Anyhow, it could be assumed that the therapeutic effectiveness of the present study might have come from the secondary elevation of IGF-1 by rhGH administration.

The benefits were also observable in the changes of DTT. Referring to the result of the control group, which showed an obvious decrement of the fibers and only 1 showed a minimal increment, the GH group showed relatively less fiber loss. Morever, 1 case showed an obvious increment of the fibers. A fMRI study revealed persistent additional activations in fMRI images of patients with complete recovery. ${ }^{40}$ Similarly, our patient who underwent an fMRI showed additional area of activation, during the affected hand grasp task. It might be interpreted that his brain recruited additional cortical areas, adjacent to the primary lesion for conducting the same task.

The current study had limitations. The subject number was small and it proceeded in the open-labeled study. Therefore, whether the GH therapy can exactly facilitate the regeneration of the nerve cells with increasing serum level of IGF-1, which could not be determined in our study. To explore the effect of GH treatment, studies with larger sample size, longterm IGF-1 follow up and doubleblind study are needed to be conducted. However, the present study has significance as the first pilot study to explore the effects of GH treatment for completed stroke patients, and to reveal no adverse effects in GH treatment. The role of IGF-1 in the GH therapy should be enlightened through further studies. Another limitation is an uneven distribution of the K-MBI scores in the control group. K-MBI score of one of the control patient was too high for the improvement by rehabilitation therapy. However, because the scores of other evaluations did not show any changes, and even showed a decrease, the KMBI scores were also expected to show little significant changes.

\section{CONCLUSION}

The rhGH (Declage ${ }^{\circledR}$ ) seemed to be safe and effective in the treatment of patients with completed stroke patients in active rehabilitation with fatigue reducing effects. 
DTT and fMRI studies partially supported the benefits of rhGH therapy. In a long-term follow-up, rhGH (Declage ${ }^{\circledR}$ ) seemed to be safe in completed stroke patients without any side effects. Further studies with larger population are needed.

\section{ACKNOWLEDGEMENTS}

This work was supported by LG Life Sciences Ltd. There are no conflicts of interest.

\section{REFERENCES}

1. Genton E, Bamett HJ, Fields WS, Gent M, Hoak JC. XIV. Cerebral ischemia: the role of thrombosis and of antithrombotic therapy. Study group on antithrombotic therapy. Stroke 1977; 8: 150-175

2. Johansson BB. Current trends in stroke rehabilitation. A review with focus on brain plasticity. Acta Neurol Scand 2011; 123: 147-159

3. Cramer SC, Riley JD. Neuroplasticity and brain repair after stroke. Curr Opin Neurol 2008; 21: 76-82

4. Jones TA, Schallert T. Overgrowth and pruning of dendrites in adult rats recovering from neocortical damage. Brain Res 1992; 581: 156-160

5. Rossini PM, Calautti C, Pauri F, Baron JC. Post-stroke plastic reorganisation in the adult brain. Lancet Neurol 2003; 2: 493-502

6. Parent JM, Vexler ZS, Gong C, Derugin N, Ferriero DM. Rat forebrain neurogenesis and striatal neuron replacement after focal stroke. Ann Neurol 2002; 52: 802-813

7. Soriano MA, Tessier M, Certa U, Gill R. Parallel gene expression monitoring using oligonucleotide probe arrays of multiple transcripts with an animal model of focal ischemia. J Cereb Blood Flow Metab 2000; 20: 1045-1055

8. Lu XC, Williams AJ, Yao C, Berti R, Hartings JA, Whipple R, Vahey MT, Polavarapu RG, Woller KL, Tortella FC, et al. Microarray analysis of acute and delayed gene expression profile in rats after focal ischemic brain injury and reperfusion. J Neurosci Res 2004; 77: 843-857

9. Yan YP, Sailor KA, Vemuganti R, Dempsey RJ. Insulinlike growth factor- 1 is an endogenous mediator of focal ischemia-induced neural progenitor proliferation.
Eur J Neurosci 2006; 24: 45-54

10. Bath KG, Lee FS. Neurotrophic factor control of adult SVZ neurogenesis. Dev Neurobiol 2010; 70: 339-349

11.Zheng WH, Quirion R. Comparative signaling pathways of insulin-like growth factor- 1 and brain-derived neurotrophic factor in hippocampal neurons and the role of the PI3 kinase pathway in cell survival. J Neurochem 2004; 89: 844-852

12. Denti L, Annoni V, Cattadori E, Salvagnini MA, Visioli S, Merli MF, Corradi F, Ceresini G, Valenti G, Hoffman $\mathrm{AR}$, et al. Insulin-like growth factor 1 as a predictor of ischemic stroke outcome in the elderly. Am J Med 2004; 117: 312-317

13. Beilharz EJ, Russo VC, Butler G, Baker NL, Connor B, Sirimanne ES, Dragunow M, Werther GA, Gluckman $\mathrm{PD}$, Williams CE, et al. Co-ordinated and cellular specific induction of the components of the IGF/IGFBP axis in the rat brain following hypoxic-ischemic injury. Brain Res Mol Brain Res 1998; 59: 119-134

14. Guan J, Bennet L, Gluckman PD, Gunn AJ. Insulin-like growth factor-1 and post-ischemic brain injury. Prog Neurobiol 2003; 70: 443-462

15. O'Kusky JR, Ye P, D'Ercole AJ. Insulin-like growth factor-I promotes neurogenesis and synaptogenesis in the hippocampal dentate gyrus during postnatal development. J Neurosci 2000; 20: 8435-8442

16. D'Ercole AJ, Ye P, O'Kusky JR. Mutant mouse models of insulin-like growth factor actions in the central nervous system. Neuropeptides 2002; 36: 209-220

17. Heck S, Lezoualc'h F, Engert S, Behl C. Insulin-like growth factor-1-mediated neuroprotection against oxidative stress is associated with activation of nuclear factor kappaB. J Biol Chem 1999; 274: 9828-9835

18. Matsuzaki H, Tamatani M, Mitsuda N, Namikawa K, Kiyama H, Miyake S, Tohyama M. Activation of Akt kinase inhibits apoptosis and changes in Bcl-2 and Bax expression induced by nitric oxide in primary hippocampal neurons. J Neurochem 1999; 73: 2037-2046

19. Delaney CL, Russell JW, Cheng HL, Feldman EL. Insulin-like growth factor-I and over-expression of BclxL prevent glucose-mediated apoptosis in Schwann cells. J Neuropathol Exp Neurol 2001; 60: 147-160

20. Ness JK, Scaduto RC Jr, Wood TL. IGF-I prevents glutamate-mediated bax translocation and cytochrome $\mathrm{C}$ release in $\mathrm{O} 4+$ oligodendrocyte progenitors. Glia 2004; 46: 183-194 
21. Mason JL, Jones JJ, Taniike M, Morell P, Suzuki K, Matsushima GK. Mature oligodendrocyte apoptosis precedes IGF-1 production and oligodendrocyte progenitor accumulation and differentiation during demyelination/remyelination. J Neurosci Res 2000; 61: 251-262

22. Liu XF, Fawcett JR, Hanson LR, Frey WH 2nd. The window of opportunity for treatment of focal cerebral ischemic damage with noninvasive intranasal insulinlike growth factor-I in rats. J Stroke Cerebrovasc Dis 2004; 13: 16-23

23. Bach LA. The insulin-like growth factor system: towards clinical applications. Clin Biochem Rev 2004; 25: $155-164$

24. Renehan AG, Zwahlen M, Minder C, O'Dwyer ST, Shalet SM, Egger M. Insulin-like growth factor (IGF)-I, IGF binding protein-3, and cancer risk: systematic review and meta-regression analysis. Lancet 2004; 363 : 1346-1353

25. Russo VC, Gluckman PD, Feldman EL, Werther GA. The insulin-like growth factor system and its pleiotropic functions in brain. Endocr Rev 2005; 26: 916-943

26. Rudman D, Feller AG, Nagraj HS, Gergans GA, Lalitha PY, Goldberg AF, Schlenker RA, Cohn L, Rudman IW, Mattson DE. Effects of human growth hormone in men over 60 years old. N Engl J Med 1990; 323: 1-6

27. Schabitz WR, Hoffmann TT, Heiland S, Kollmar R, Bardutzky J, Sommer C, Schwab S. Delayed neuroprotective effect of insulin-like growth factor-i after experimental transient focal cerebral ischemia monitored with mri. Stroke 2001; 32: 1226-1233

28. Endres M, Piriz J, Gertz K, Harms C, Meisel A, Kronenberg G, Torres-Aleman I. Serum insulin-like growth factor I and ischemic brain injury. Brain Res 2007; 1185: 328-335

29. Chopp M, Zhang ZG, Jiang Q. Neurogenesis, angiogenesis, and MRI indices of functional recovery from stroke. Stroke 2007; 38 Suppl 2: 827-831

30. Heller SL, Heier LA, Watts R, Schwartz TH, Zelenko N, Doyle W, Devinsky O. Evidence of cerebral reorgani- zation following perinatal stroke demonstrated with fMRI and DTI tractography. Clin Imaging 2005; 29: 283-287

31. Rocca MA, Filippi M. Functional MRI to study brain plasticity in clinical neurology. Neurol Sci 2006; 27 Suppl 1: S24-26

32. Harman SM, Blackman MR. Use of growth hormone for prevention or treatment of effects of aging. J Gerontol A Biol Sci Med Sci 2004; 59: 652-658

33. Levy RM. Growth hormone therapy in adults and children. N Engl J Med 2000; 342: 359

34. Perls TT, Reisman NR, Olshansky SJ. Provision or distribution of growth hormone for "antiaging": clinical and legal issues. JAMA 2005; 294: 2086-2090

35. Pathipati P, Surus A, Williams CE, Scheepens A. Delayed and chronic treatment with growth hormone after endothelin-induced stroke in the adult rat. Behav Brain Res 2009; 204: 93-101

36. Ziv Y, Finkelstein A, Geffen Y, Kipnis J, Smirnov I, Shpilman S, Vertkin I, Kimron M, Lange A, Hecht T, et al. A novel immune-based therapy for stroke induces neuroprotection and supports neurogenesis. Stroke 2007; 38 Suppl 2: 774-782

37.Zhu W, Fan Y, Frenzel T, Gasmi M, Bartus RT, Young WL, Yang GY, Chen Y. Insulin growth factor-1 gene transfer enhances neurovascular remodeling and improves long-term stroke outcome in mice. Stroke 2008; 39: 1254-1261

38. Zhang WJ, Tan YF, Yue JT, Vranic M, Wojtowicz JM. Impairment of hippocampal neurogenesis in streptozotocin-treated diabetic rats. Acta Neurol Scand 2008; 117: 205-210

39. Lupien SB, Bluhm EJ, Ishii DN. Systemic insulin-like growth factor-I administration prevents cognitive impairment in diabetic rats, and brain IGF regulates learning/memory in normal adult rats. J Neurosci Res 2003; 74: 512-523

40. Krakauer JW. Functional imaging of motor recovery after stroke: remaining challenges. Curr Neurol Neurosci Rep 2004; 4: 42-46 